\title{
Fractional-Order Modeling and Analysis of a Variable Structure Hybrid Energy Storage System for EVs
}

\author{
Jianlin Wang $\mathbb{D},{ }^{1}$ Dan Xu $\mathbb{D}^{2},{ }^{2}$ Jiahui Zhou, ${ }^{2}$ and Jinlu $\mathrm{Mao}^{2}$ \\ ${ }^{1}$ School of Electrical Information Engineering, North Minzu University, Yinchuan, NingXia, China \\ ${ }^{2}$ School of Mechanical Engineering, Xi'an Jiaotong University, Xi'an, Shaanxi, China \\ Correspondence should be addressed to Dan Xu; xudan@xjtu.edu.cn
}

Received 16 June 2020; Revised 12 November 2020; Accepted 25 November 2020; Published 9 December 2020

Academic Editor: Shuping He

Copyright (C) 2020 Jianlin Wang et al. This is an open access article distributed under the Creative Commons Attribution License, which permits unrestricted use, distribution, and reproduction in any medium, provided the original work is properly cited.

Hybrid energy storage system has been widely studied as an important technology for electric vehicles. Since the hybrid energy storage system is a nonlinear and complex system, the modeling of the system and the high-precision nonlinear control strategy are technical difficulties for research. The establishment of a high-precision mathematical model of the hybrid energy storage system is the basis for the study of high-quality nonlinear control algorithms. Fortunately, the theory of fractional calculus can help build accurate mathematical models of hybrid energy storage systems. In order to obtain the high-quality nonlinear control strategy of this complex system, this paper, respectively, carried out fractional-order modeling and analysis on the three basic equivalent working states of the hybrid energy storage system of electric vehicles. Among them, the fractional-order average state space model is carried out for the equivalent Buck and Boost mode. Also, the steady-state analysis of the equivalent Dual-Boost mode is carried out by combining the fractional-order calculus theory with the equivalent small parameter variable method. Finally, the effectiveness and precision of the fractional-order model are proved by simulation and experiment.

\section{Introduction}

The hybrid energy storage system (HESS) of electric vehicles, that is, the composite power supply technology, is a combination of two or more energy sources to form a power system for electric vehicles $[1,2]$. Generally, the overall performance of electric vehicles is improved by complementing the advantages of various energy sources, which is a relatively advanced technology in the field of electric vehicles. As we all know, the hybrid energy storage system contains two or more power supplies, and there are also multiple DC-DC converters with strong nonlinear characteristics, which make the design of the control strategy for the hybrid energy storage system difficult. In order to be able to design high-performance control strategies, it is necessary to carry out topology design and high-precision system modeling for hybrid energy storage systems.

The topology of a HESS is generally passive, semiactive, active, or cascaded. It is all well known, and each topology of HESS has obvious advantages and disadvantages [3]. In order to integrate the advantages of various topologies, a new variable structure HESS has been proposed, which can realize the function of battery/supercapacitor (SC) semiactive structure, SC/battery semiactive structure, and fully active structure. The topology of the new variable structure HESS is shown in Figure 1. It mainly has three working states; that is, it works in the Buck mode when the SC absorbs the braking energy; it works in the Boost mode when the SC or the battery is outputting separately; and it works in the Dual-Boost mode when the SC and the battery are outputting together.[4, 5].

The control problem of this nonlinear complex system is a difficult point, but there are some research results that can be used. For example, the sliding mode control method is used to control the DC-DC converter to implement power distribution, and the convergence time of the positive Markov neural network is optimized [6-8], and the fractional calculus theory is used to perform fractional control to obtain high performance [9]. However, the accuracy of these control strategies needs to be based on a high-precision 
system model, and the design of HESS for electric vehicles is diverse so that there are relatively few studies on the modeling of variable structure HESS for electric vehicles.

An accurate mathematical model for the equivalent DCDC converter system corresponding to each working state is the basis for applying the variable structure HESS use in electric vehicles. Considering the nondualities of commercial inductors, it is difficult to describe such circuit components with long-memory characteristics using conventional integerorder calculus. Fortunately, the fractional-order theory is gradually maturing, and in the past decade, fractional-order calculus has been applied in almost all fields of science, engineering, and mathematics. Concepts from fractional-order circuits and systems have attracted much attention from the electrical engineering community. Some research results prove that the fractional-order model has higher precision than the integer-order model in mathematical modeling of nonlinear circuit systems, and the integer-order model is a special case of the fractional-order model [10]. Therefore, this article focuses on the fractional-order modeling research on the three working states of the variable structure HESS.

The volt-ampere characteristics of fractional inductor and capacitor shown in Figure 1 can be described as

$$
\begin{aligned}
& v_{L 1}=L \frac{d^{\alpha} i_{L 1}}{\mathrm{~d} t^{\alpha},} \\
& v_{L 2}=L \frac{d^{\beta} i_{L 2}}{\mathrm{~d} t^{\beta}}, \\
& i_{C}=C \frac{d^{\gamma} v_{C}}{\mathrm{~d} t^{\gamma}},
\end{aligned}
$$

where $\alpha$ is the order of the fractional inductor $L_{1}, \beta$ is the order of the fractional inductor $L_{2}$, and $\gamma$ is the order of the fractional capacitor $C$, respectively [11].

In this article, the fractional-order modeling of equivalent DC-DC converters corresponding to the three working states of the variable structure HESS is mainly based on the fractional-order characteristics of inductors and capacitors. The rest of this manuscript is organized as follows: Section 2 introduces the fractional-order modeling of the equivalent Buck converter for braking energy recovery. Section 3 introduces the fractional-order modeling of the equivalent Boost converter for power output. Section 4 introduces the steady-state analysis of the dual-Boost output mode based on the fractional-order equivalent small parameter variable method. In Section 5, simulations and experiments are performed to verify the fractional-order mode. Finally, conclusions are given in Section 6.

\section{Fractional-Order Modeling of Equivalent Buck Converter for Braking Energy Recovery}

From the working principle of the variable structure HESS, it is known that when the electric vehicle brakes to produce energy, it is absorbed by the SC alone. In this mode of operation, the equivalent Buck converter of the variable structure HESS is shown in Figure 2. The input voltage is

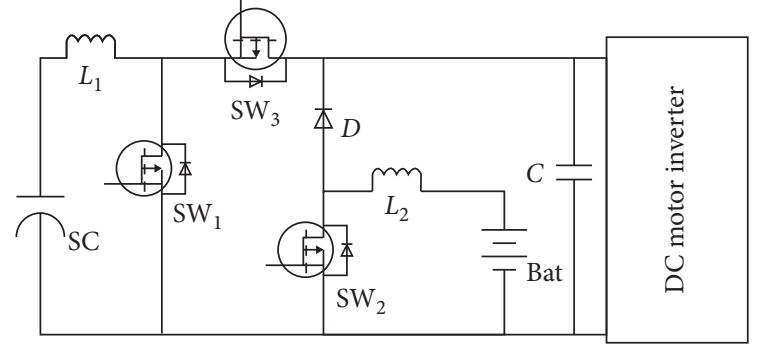

FIgURE 1: Topology of the variable structure HESS.

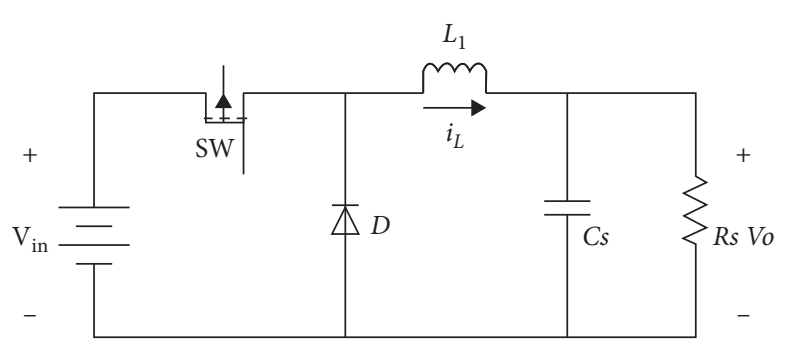

Figure 2: Equivalent Buck converter of the variable structure HESS.

equivalent to the voltage of the motor inverter, the load is the internal resistance of the SC, and the capacitance is the equivalent capacitance of the SC.

The equivalent Buck converter has two main operating states. The operating state 1 is that the switch SW is closed; that is, the capacitor is charged; the operating state 2 is that the switch SW is open; that is, the capacitor is discharged. Throughout one duty cycle period, the inductor voltage can be defined as

$$
v_{L}= \begin{cases}v_{\text {in }}-v_{o}, & 0<t<\mathrm{d} T, \\ -v_{o}, & \mathrm{~d} T<t<T .\end{cases}
$$

The average current of the inductor current over the entire period can be described as

$$
\left\langle i_{L 1}(t, \alpha)\right\rangle_{T}=\frac{1}{T} \int_{t}^{t+T} i_{L 1}(\tau, \alpha) \mathrm{d} \tau .
$$

The average inductor current also can be converted into a form by fractional-order calculation as follows:

$$
\frac{d^{\alpha}\left\langle i_{L 1}(t, \alpha)\right\rangle_{T}}{\mathrm{~d} t^{\alpha}}=\frac{1}{T} \int_{t}^{t+T}\left(\frac{d^{\alpha} i_{L 1}(t, \alpha)}{\mathrm{d} \tau^{\alpha}}\right) \mathrm{d} \tau=\left\langle\frac{d^{\alpha} i_{L 1}(t, \alpha)}{\mathrm{d} t^{\alpha}}\right\rangle
$$

where $\left\langle i_{L 1}(t, \alpha)\right\rangle$ is the superposition of the AC component under the influence of the DC component and the highfrequency switching harmonics. Similarly, the input voltage, output voltage, inductor current, and switching duty cycle in an equivalent Buck converter can be considered as a superposition of the DC component and the interference component $[12,13]$.

Assume that the equivalent capacitance of the SC also has a fractional-order characteristic, and the order is defined 
as $\rho$. A fractional-order average state space model can be established for equivalent Buck converter.

When $t \in[0, \mathrm{~d} T]$, the equation of state for the equivalent Buck converter is

$$
\begin{aligned}
& \frac{d^{\alpha}\left\langle i_{L 1}\right\rangle}{\mathrm{d} t^{\alpha}}=\frac{1}{L_{1}}\left(\left\langle v_{i n}\right\rangle-\left\langle v_{o}\right\rangle\right), \\
& \frac{d^{\rho}\left\langle v_{o}\right\rangle}{\mathrm{d} t^{\rho}}=\frac{1}{C_{s}}\left(\left\langle i_{L 1}\right\rangle-\frac{\left\langle v_{o}\right\rangle}{R_{s}}\right) .
\end{aligned}
$$

When $t \in[d T, T]$, the equation of state for the equivalent Buck converter is

$$
\begin{aligned}
& \frac{d^{\alpha}\left\langle i_{L 1}\right\rangle}{\mathrm{d} t^{\alpha}}=-\frac{1}{L_{1}}\left(\left\langle v_{o}\right\rangle\right), \\
& \frac{d^{\rho}\left\langle v_{o}\right\rangle}{\mathrm{d} t^{\rho}}=\frac{1}{C_{s}}\left(\left\langle i_{L 1}\right\rangle-\frac{\left\langle v_{o}\right\rangle}{R_{s}}\right) .
\end{aligned}
$$

Introducing the duty cycle function in equations (5) and (6), the fractional-order average state model of the equivalent Buck circuit is obtained, as

$$
\left[\begin{array}{c}
\frac{d^{\alpha}\left\langle i_{L 1}\right\rangle}{\mathrm{d} t^{\alpha}} \\
\frac{d^{\rho}\left\langle v_{o}\right\rangle}{\mathrm{d} t^{\rho}}
\end{array}\right]=\left[\begin{array}{cc}
0 & -\frac{1}{L_{1}} \\
\frac{1}{C_{s}} & -\frac{1}{R_{s} C_{s}}
\end{array}\right]\left[\begin{array}{c}
\left\langle i_{L 1}\right\rangle \\
\left\langle v_{o}\right\rangle
\end{array}\right]+\left[\begin{array}{c}
\frac{\langle d\rangle}{L_{1}} \\
0
\end{array}\right]\left\langle v_{\text {in }}\right\rangle .
$$

Based on the fractional-order Laplace transform method [6], the Laplace transform of equation (7) is obtained:

$$
\left[\begin{array}{c}
s^{\alpha}\left\langle i_{L 1}(s)\right\rangle \\
s^{\rho}\left\langle v_{o}(s)\right\rangle
\end{array}\right]=\left[\begin{array}{cc}
0 & -\frac{1}{L_{1}} \\
\frac{1}{C_{s}} & -\frac{1}{R_{s} C_{s}}
\end{array}\right]\left[\begin{array}{c}
\left\langle i_{L 1}(s)\right\rangle \\
\left\langle v_{o}(s)\right\rangle
\end{array}\right]+\left[\begin{array}{c}
\frac{\langle d(s)\rangle}{L_{1}} \\
0
\end{array}\right]\left\langle v_{\text {in }}(s)\right\rangle .
$$

The transfer function of the output voltage to the input voltage is obtained by equation (8) as

$$
G_{V}(s)=\left.\frac{v_{o}(s)}{v_{\text {in }}(s)}\right|_{\widehat{d}(s)=0}=\frac{\left(D / L_{1} C_{S}\right)}{s^{\alpha \rho}+\left(1 / R_{S} C_{S}\right) s^{\alpha}+\left(1 / L_{1} C_{S}\right)} .
$$

Assuming that the input and output voltages are constant during the cycle, we can obtain the input and output energies as

$$
\begin{aligned}
E_{\text {in }} & =v_{\text {in }} \int_{0}^{\mathrm{d} T} i_{L 1}(\tau, \alpha) \mathrm{d} \tau, \\
& =v_{\text {in }}\left[\mathrm{d} T i_{R}+\frac{\left(v_{\mathrm{in}}-v_{o}\right)}{L_{1} \Gamma(\alpha+1)} T^{\alpha+1} d\left(d^{\alpha}-d\right)\right], \\
E_{o} & =v_{o}\left(\int_{0}^{\mathrm{d} T} i_{L 1}(\tau, \alpha) \mathrm{d} \tau+\int_{\mathrm{d} T}^{T} i_{L 2}(\tau, \alpha) \mathrm{d} \tau\right), \\
& =v_{o}\left[T i_{R}+\frac{\left(d^{\alpha+1}-d\right) v_{i n}+(1-d)^{\alpha}}{L_{1} \Gamma(\alpha+2)} T^{\alpha+1}\right] .
\end{aligned}
$$

At steady state, the average energy efficiency is

$$
\eta=\frac{E_{o}}{E_{m}}=\frac{d}{\left(1-(1-d)^{\alpha}\right) i_{R}}\left[i_{R}-\frac{v_{\mathrm{in}} T^{\alpha}(1-d)^{\alpha}\left(d^{\alpha}-d\right)}{L_{1} \Gamma(\alpha+2)}\right] .
$$

\section{Fractional-Order Modeling of Equivalent Boost Converter for Power Output}

When the power demand of the electric vehicle is positive, the variable structure HESS works in the Boost mode and boosts the voltage of the battery or the SC for output. In this working state, the equivalent Boost converter of the variable structure HESS is shown in Figure 3. The input voltage $v_{\text {in }}$ is equivalent to the voltage of the battery or SC, and the load is the motor inverter. In this section, the battery boost output is chosen as an example to illustrate the fractional-order modeling and analysis of the Boost mode.

Similar to the equivalent Buck converter, the equivalent Boost converter also contains two operating states. In one duty cycle, the inductor voltage can be described as

$$
v_{L}= \begin{cases}v_{\text {in }}, & 0<t<\mathrm{d} T, \\ v_{\text {in }}-v_{o}, & \mathrm{~d} T<t<T .\end{cases}
$$

When $t \in[0, \mathrm{~d} T]$, the equation of state for the equivalent Boost converter is

$$
\begin{aligned}
& \frac{d^{\beta}\left\langle i_{L 2}\right\rangle}{\mathrm{d} t^{\beta}}=\frac{1}{L_{2}}\left\langle v_{\mathrm{in}}\right\rangle, \\
& \frac{d^{\gamma}\left\langle v_{o}\right\rangle}{\mathrm{d} t^{\gamma}}=\frac{1}{R C}\left\langle v_{o}\right\rangle .
\end{aligned}
$$

When $t \in[\mathrm{d} T, T]$, the equation of state for the equivalent Boost converter is

$$
\begin{aligned}
& \frac{d^{\beta}\left\langle i_{L 2}\right\rangle}{\mathrm{d} t^{\beta}}=\frac{1}{L_{2}}\left(\left\langle v_{i n}\right\rangle-\left\langle v_{o}\right\rangle\right), \\
& \frac{d^{\gamma}\left\langle v_{o}\right\rangle}{\mathrm{d} t^{\gamma}}=\frac{1}{C}\left(\left\langle i_{L 2}\right\rangle-\frac{\left\langle v_{o}\right\rangle}{R}\right) .
\end{aligned}
$$

Introducing the duty cycle function in equations (13) and (14), the fractional-order average state model of the equivalent Buck circuit is obtained, as

$$
\left[\begin{array}{c}
\frac{d^{\beta}\left\langle i_{L}\right\rangle}{\mathrm{d} t^{\beta}} \\
\frac{d^{\gamma}\left\langle v_{o}\right\rangle}{\mathrm{d} t^{\gamma}}
\end{array}\right]=\left[\begin{array}{cc}
0 & -\frac{1-\langle d\rangle}{L_{2}} \\
\frac{1-\langle d\rangle}{C} & -\frac{1}{R C}
\end{array}\right]\left[\begin{array}{c}
\left\langle i_{L 2}\right\rangle \\
\left\langle v_{o}\right\rangle
\end{array}\right]+\left[\begin{array}{c}
\frac{1}{L_{2}} \\
0
\end{array}\right]\left\langle v_{\text {in }}\right\rangle .
$$

Based on the fractional-order Laplace transform method, the Laplace transform of equation (15) is obtained: 


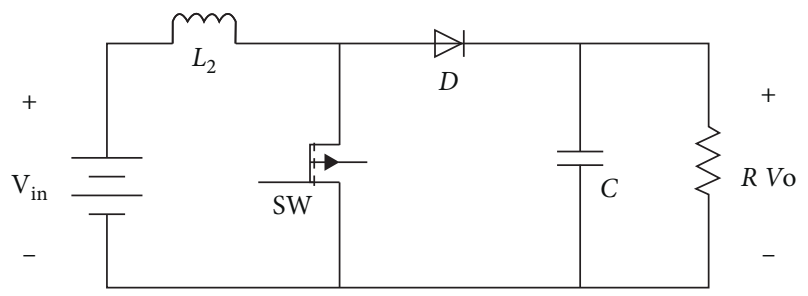

Figure 3: Equivalent Boost converter of the variable structure HESS.

$$
\left[\begin{array}{c}
s^{\beta}\left\langle i_{L 2}(s)\right\rangle \\
s^{\gamma}\left\langle v_{o}(s)\right\rangle
\end{array}\right]=\left[\begin{array}{cc}
0 & -\frac{1-\langle d(s)\rangle}{L_{2}} \\
\frac{1-\langle d(s)\rangle}{C} & -\frac{1}{R C}
\end{array}\right]\left[\begin{array}{c}
\left\langle i_{L 2}(s)\right\rangle \\
\left\langle v_{o}(s)\right\rangle
\end{array}\right]+\left[\begin{array}{c}
\frac{1}{L_{2}} \\
0
\end{array}\right]\left\langle v_{\text {in }}(s)\right\} .
$$

The transfer function of the output voltage to the input voltage is obtained by equation (16) as

$$
G_{V}(s)=\left.\frac{\left\langle v_{o}(s)\right\rangle}{\left\langle v_{i n}(s)\right\rangle}\right|_{\widehat{d}(s)=0}=\frac{\left(1-D / L_{2} C\right)}{s^{\beta+\gamma}+(1 / R C) s^{\beta}+\left((1-D)^{\beta} / L C\right)} .
$$

Assuming that the input and output voltages are constant during the cycle, we can obtain the input and output energies as

$$
\begin{aligned}
E_{\text {in }} & =v_{\text {in }}\left[\int_{0}^{\mathrm{d} T} i_{L 2}^{\prime}(\tau, \beta) \mathrm{d} \tau+\int_{a T}^{T} i_{L 2}^{\prime \prime}(\tau, \beta) \mathrm{d} \tau\right], \\
& =\frac{v_{\text {in }} T}{1-d}\left[i_{R}-\frac{v_{\text {in }} T^{\beta}\left(1-d+d^{2}-d^{\beta+1}\right)-v_{o} T^{\beta}(1-d)^{\beta+1}}{L_{2} \Gamma(\beta+2)}\right], \\
E_{o} & =v_{o} \int_{a T}^{T} i_{L 2}^{\prime \prime}(\tau, \beta) \mathrm{d} \tau=v_{o} i_{R} T=\frac{v_{\text {in }} i_{R} T}{1-d^{\beta}} .
\end{aligned}
$$

At steady state, the average energy efficiency is

$$
\eta=\frac{E_{o}}{E_{\text {in }}}=\frac{1-d^{\beta}}{1-d}\left[1-\frac{v_{\text {in }} T^{\beta}\left(1-d+d^{2}-d^{\beta+1}\right)-v_{o}(1-d)^{\beta+1}}{L_{2} \Gamma(\beta+2) i_{R}}\right] .
$$

\section{Fractional-Order Modeling and Steady-State Analysis for Dual-Boost Output Mode}

When the state of the battery and the SC are not in an extreme state, the variable structure HESS is often operating in a common output mode. In this mode, the switch SW3 is turned off, and the variable structure HESS can be equivalent to the dual-Boost parallel output. The equivalent DC-DC converter is shown in Figure 4. Such a parallel output circuit system has high requirements for the design of the control algorithm and the implementation of the power allocation strategy.

The state space averaging has many advantages in modeling, but not enough precision. It cannot perform steady-state analysis of this type of circuitry, especially ripple analysis. The equivalent small parameter variable method is

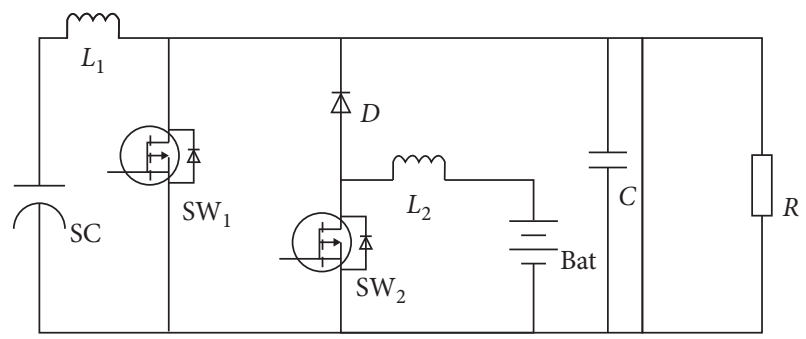

FIgURE 4: Equivalent dual-Boost converter of variable structure HESS.

a high-precision symbol analysis method combining the disturbance analysis method and the harmonic balance method [4]. This method does not need to introduce small parameter quantities, which has certain advantages for the ripple analysis of nonlinear high-order systems $[14,15]$.

Considering the fractional-order characteristics of the capacitor and inductor in the circuit system shown in Figure 4 , the results of the circuit analysis are

$$
\left\{\begin{array}{l}
\frac{d^{\alpha} i_{L 1}}{\mathrm{~d} t^{\alpha}}=\frac{V_{S C}}{L_{1}}-\frac{v_{C}}{L_{1}}, \\
\frac{d^{\beta} i_{L 2}}{\mathrm{~d} t^{\beta}}=\frac{V_{\text {Bat }}}{L_{2}}-\frac{v_{C}}{L_{2}}, \\
\frac{d^{\gamma} v_{C}}{\mathrm{~d} t^{\gamma}}=\frac{\left(i_{L_{1}}+i_{L_{2}}\right)}{C}-\frac{v_{C}}{R} .
\end{array}\right.
$$

The state equation of the dual-PWM switch Boost equivalent circuit system is described as

$$
G_{0}\left(p^{\alpha} \quad p^{\beta} \quad p^{\gamma}\right) x+G_{1} f^{(1)}(x)+G_{2} f^{(2)}(x)=U,
$$

where $x=\left[\begin{array}{lll}i_{L 1} & i_{L 2} & v_{C}\end{array}\right]^{T}, \quad G_{0}=\left[\begin{array}{ccc}p^{\alpha} & 0 & \left(1 / L_{1}\right) \\ 0 & p^{\beta} & \left(1 / L_{2}\right) \\ -(1 / C) & -(1 / C) & p^{\gamma}+(1 / R C)\end{array}\right]$, $G_{1}=\left[\begin{array}{ccc}0 & 0 & -\left(1 / L_{1}\right) \\ 0 & 0 & 0 \\ (1 / C) & 0 & 0\end{array}\right], G_{2}=\left[\begin{array}{ccc}0 & 0 & 0 \\ 0 & 0 & -\left(1 / L_{2}\right) \\ 0 & (1 / C) & 0\end{array}\right], U=$ $\left[\begin{array}{c}\left(V_{S C} / L_{1}\right) \\ \left(V_{\mathrm{Bat}} / L_{2}\right) \\ 0\end{array}\right], p^{\alpha}=D^{\alpha}$ is fractional calculus operator, $f^{(1)}(x)=\delta^{(1)}(t) x, f^{(2)}(x)=\delta^{(2)}(t) x$, and $\delta(t)$ is the pulse function.

State variable $x$ and $\delta(t)$ pulse function are, respectively, expanded into a series form of a sum of a main component and a small component, that is,

$$
\begin{gathered}
x=x_{0}+\sum_{i=1}^{\infty} \varepsilon^{i} x_{i}, \\
\delta^{(n)}(t)=\delta_{0}^{(n)}+\sum_{i=1}^{\infty} \varepsilon^{i} \delta_{i}^{(n)} .
\end{gathered}
$$

Substituting equations (22) and (23) into equation (21), we get 


$$
f^{(n)}\left(\delta^{(n)}, x\right)=f_{0}^{(n)}+\sum_{i=1}^{\infty} \varepsilon^{i} f_{i}^{(n)}
$$

where $i$ is the order of the harmonics and $x_{i}, \delta_{i}$, and $f_{i}$ are the $i$-th order components of $x, \delta(x)$, and $f(x) . \varepsilon$ is a small component symbol, which only means that $x_{i}$ is much smaller than $x$.

The Fourier series of $x_{0}$ and $x_{i(i \geq 1)}$ are expanded to

$$
\begin{aligned}
& x_{0}=\sum_{k \in E_{0}} x_{k 0}=a_{00}, \\
& x_{i}=\sum_{k \in E_{i r}} x_{k j}=a_{0 i}+\sum_{k \in E_{i r}}\left[a_{k i} e^{j k \tau}+\bar{a}_{k i} e^{-j k \tau}\right],
\end{aligned}
$$

where $\tau=\omega t=(2 \pi t / T), a_{0 t}$ is the DC component of $x_{i}$, and $a_{k i}$ is the $k$-th order amplitude of the $i$-th harmonic. $\left\{E_{0}\right\}$ is the spectrum set of the state vector $x_{0}$, and $\left\{E_{i r}\right\}$ is the spectrum set of the correction $x_{i}$ during the iterative calculation.

Furthermore, the Fourier series expansion of the $\delta_{0}^{(n)}$ and $\delta_{i(i \geq 1)}^{(n)}$ is

$$
\begin{aligned}
& \delta_{0}^{(n)}=b_{0}^{(n)}+b_{1}^{(n)} e^{j r}+\bar{b}_{1}^{(n)} e^{-j t}, \\
& \delta_{i}^{(n)}=b_{2 i}^{(n)} e^{j 2 i \tau}+b_{2 i+1}^{(n)} e^{j(2 i+1) \tau}+\bar{b}_{2 i}^{(n)} e^{-j 2 i \tau}+\bar{b}_{2 i+1}^{(n)} e^{-j(2 i+1) \tau},
\end{aligned}
$$

where

$b_{0}^{(n)}=1 / T \int_{0}^{T} \delta^{(n)}(t) \mathrm{d} t=d^{(n)}$ and $b_{i}^{(n)}=1 / 2\left(a_{i}^{(n)}-j \beta_{i}^{(n)}\right)$.

Furthermore, equation (20) can be rewritten as

$$
\left\{\begin{array}{l}
f_{0}^{(n)}=\delta_{0}^{(n)} x_{0}, \\
f_{1}^{(n)}=\delta_{0}^{(n)} x_{1}+\delta_{1}^{(n)} x_{0}, \\
f_{2}^{(n)}=\delta_{0}^{(n)} x_{2}+\delta_{1}^{(n)} x_{1}+\delta_{2}^{(n)} x_{0}, \\
\cdots
\end{array}\right.
$$

Expand the function $f_{i}^{(n)}(x)$ into the series form of the sum of the main and small components, which can be expressed as

$$
f_{i}^{(n)}=f_{i=1}^{(n)}+\varepsilon R_{i+1}, \quad(i=0,1,2, \ldots,) .
$$

When $i=0, f_{0 m}^{(n)}$ is the main component of $f_{0}^{(n)}$, which is the same as the spectrum of $x_{0}$, and $R_{1}$ is the remaining high-order small components. According to the spectrum of $R_{1}$, the frequency set of can be determined. Similarly, the frequency set of $f_{0 m}^{(n)}$ can be determined according to the spectrum of $f_{i m}^{(n)} . f_{0 m}^{(n)}, f_{i m}^{(n)}$, and $R_{i}$ in equation (28) are

$$
\begin{aligned}
f_{0 m}^{(n)} & =h_{00}+\sum_{k \in E_{0}}\left(h_{\Sigma 0} e^{j k \tau}+\bar{h}_{k 0} e^{-j k z}\right), \\
f_{i m}^{(n)} & =h_{0 i}+\sum_{k \in E_{i r}}\left(h_{k i} e^{j k r}+\bar{h}_{k i} e^{-j k \tau}\right), \\
R_{i} & =g_{0 i}+\sum_{k \in E_{i r}}\left(g_{k i} e^{j k \tau}+\bar{g}_{k i} e^{-j k \tau}\right),
\end{aligned}
$$

where $h_{00}, h_{0 i}$, and $g_{0 i}$ are all DC components. Substitute the above equation into equation (28) to get the following equation:

$$
f^{(n)}=\left(f_{0 m}^{(n)}+\varepsilon f_{1 m}^{(n)}+\varepsilon^{2} f_{2 m}^{(n)}+\ldots\right)+\left(\varepsilon R_{1}+\varepsilon^{2} R_{2}+\ldots\right) .
$$

According to the above analysis, the principal component of the steady-state solution is DC, and the principal component of the state variable is defined as

$$
x_{0}=a_{00}=\left[I_{L_{1} 00} I_{L_{2} 00} V_{C 00}\right]^{T} .
$$

Considering that there is no derivative change in the DC component of the system, let $G_{00}=G_{0}\left(\begin{array}{ccc}0^{\alpha} & 0^{\beta} & 0^{\gamma}\end{array}\right)$, and we can get

$$
\left[\begin{array}{ccc}
0 & 0 & \frac{1-d^{(1)}}{L_{1}} \\
0 & 0 & \frac{1-d^{(2)}}{L_{2}} \\
-\frac{1-d^{(1)}}{C} & -\frac{1-d^{(2)}}{C} & \frac{1}{R C}
\end{array}\right] \cdot\left[\begin{array}{c}
I_{L_{1} \infty} \\
I_{L_{2} 00} \\
V_{C 00}
\end{array}\right]=\left[\begin{array}{c}
\frac{V_{S C}}{L_{1}} \\
\frac{V_{\text {Bat }}}{L_{2}} \\
0
\end{array}\right] .
$$

Solving equation (32) can obtain the steady-state solution of the main oscillation component, and we can get

$$
\left\{\begin{array}{l}
V_{C 00}=\frac{V_{S C}}{1-d^{(1)}}=\frac{V_{\mathrm{Bat}}}{1-d^{(2)}} \\
I_{L, 00}=\frac{V_{S C}}{2 R\left(1-d^{(1)}\right)^{2}} \\
I_{L_{2} 00}=\frac{V_{\mathrm{Bat}}}{2 R\left(1-d^{(2)}\right)^{2}}
\end{array}\right.
$$

The first-order correction contains only the fundamental component, so suppose

$$
x_{1}=a_{11} e^{j \tau}+\bar{a}_{11} e^{-j \tau}
$$

where $a_{11}=\left[\begin{array}{lll}I_{L_{1} 11} & I_{L_{2} 11} & V_{C 11}\end{array}\right]^{T}$ and $I_{L_{1} 11}, I_{L_{2} 11}$, and $V_{C 11}$ are the first-order amplitude small components of the first harmonics of $i_{L_{1} 11}, i_{L_{2} 11}$, and $v_{C 11}$, respectively. Substituting equation (34) into equation (30), we get

$$
\begin{aligned}
& f_{1 m}^{(1)}=b_{0}^{(1)}\left(a_{11} e^{j \tau}+\bar{a}_{11} e^{-j \tau}\right), \\
& f_{1 m}^{(2)}=b_{0}^{(2)}\left(a_{11} e^{j \tau}+\bar{a}_{11} e^{-j \tau}\right), \\
& R_{1}^{(1)}=a_{11}\left(b_{1}^{(1)} e^{j \tau}+\bar{b}_{1}^{(1)} e^{-j \tau}\right), \\
& R_{1}^{(2)}=a_{11}\left(b_{1}^{(2)} e^{j \tau}+\bar{b}_{1}^{(2)} e^{-j \tau}\right) .
\end{aligned}
$$


According to the calculation principle of fractional calculus, $\quad p^{\alpha} e^{j \omega t}=(j \omega)^{\alpha} e^{j \omega t}, \quad p^{\beta} e^{j \omega t}=(j \omega)^{\beta} e^{j \omega t}, \quad$ and $p^{\gamma} e^{j \omega t}=(j \omega)^{\gamma} e^{j \omega t}$. [16] The first-order correction amount that can be calculated is

$$
\left\{\begin{array}{l}
V_{C 11} \approx \frac{b_{1}\left((j \omega)^{\alpha} L_{1} I_{L, 00}+(j \omega)^{\beta} L_{2} I_{L_{2} 00}-\left(1-d^{\prime}\right) V_{C 00}\right)}{\Delta(j \omega)} \\
I_{L_{11}}=\frac{b_{1} V_{C 00}-\left(1-d^{(1)}\right) V_{C 11}}{(j \omega)^{\alpha} L_{1}} \\
I_{L_{2} 11}=\frac{b_{1} V_{C 00}-\left(1-d^{(2)}\right) V_{C 11}}{(j \omega)^{\beta} L_{2}}
\end{array}\right.
$$

According to the above calculation principle, $E_{\text {ir }}=\{0,2,3\}$ is known; then, we suppose

$$
x_{2}=a_{02}+\left(a_{22} e^{j 2 \tau}+\bar{a}_{22} e^{-j 2 \tau}+a_{32} e^{j 3 \tau}+\bar{a}_{32} e^{-j 3 \tau}\right),
$$

where $a_{02}=\left[\begin{array}{lll}I_{L_{1} 02} & I_{L_{2} 02} & V_{C 02}\end{array}\right]^{T}$ is the second-order correction of the DC component and $a_{22}=\left[\begin{array}{lll}I_{L_{1} 22} & I_{L_{2} 22} & V_{C 22}\end{array}\right]^{T}$ and $a_{32}=\left[\begin{array}{lll}I_{L_{1} 32} & I_{L_{2} 32} & V_{C 32}\end{array}\right]^{T}$ are the higher-order components of the second harmonic, respectively.

Substituting equation (37) into equation (30), after ignoring the high-order small component of the second harmonic, we can get

$$
\begin{aligned}
f_{2 m}^{(1)} \approx & b_{0}^{(1)} a_{02}+\left(b_{0}^{(1)} a_{22}+b_{3}^{(1)} \bar{a}_{11}\right) e^{j 2 \tau} \\
& +\left(b_{0}^{(1)} a_{32}+b_{1}^{(1)} a_{22}+b_{2}^{(1)} a_{11}\right) e^{j 3 t}+c . c, \\
f_{2 m}^{(2)} \approx & b_{0}^{(2)} a_{02}+\left(b_{0}^{(2)} a_{22}+b_{3}^{(2)} \bar{a}_{11}\right) e^{j 2 t} \\
& +\left(b_{0}^{(2)} a_{32}+b_{1}^{(2)} a_{22}+b_{2}^{(2)} a_{11}\right) e^{j 3 t}+\text { c.c. }
\end{aligned}
$$

Furthermore, the second-order correction of the DC component and the higher-order component of the secondorder harmonic can be obtained:

$$
\begin{aligned}
& \left\{\begin{array}{l}
V_{C 02}=\frac{\left(\bar{b}_{1} V_{C 11}+b_{1} \bar{V}_{C 11}\right)}{\left(1-d^{\prime}\right)}, \\
I_{L_{1} 02}=\frac{V_{C 02}}{2 R\left(1-d^{(1)}\right)}, \\
I_{L_{2} 02}=\frac{2 V_{C 02}}{2 R\left(1-d^{(2)}\right)}, \\
\left\{\begin{array}{l}
V_{C 22}=\frac{(1-d)\left(b_{1} V_{C 11}+b_{2} V_{C 00}+b_{3} \bar{V}_{C 11}\right)}{\Delta(j 2 \omega)}- \\
\frac{(j 2 \omega)^{\alpha} L_{1}\left(b_{1} I_{L_{1} 11}+b_{2} I_{L_{1} 00}+b_{3} \bar{I}_{L_{1} 11}\right)+(j 2 \omega)^{\beta} L_{2}\left(b_{1} I_{L_{2} 11}+b_{2} I_{L_{2} 00}+b_{3} \bar{I}_{L_{2} 11}\right)}{\Delta(j 2 \omega)} \\
I_{L_{2} 32}=\frac{\left(b_{1} V_{C 22}+b_{2} V_{C 11}+b_{3} \bar{V}_{C 00}\right)-\left(1-d^{(2)}\right) V_{C 23}}{(j 3 \omega)^{\beta} L_{2}} \\
I_{L_{1} 22}=\frac{\left(b_{1} V_{C 11}+b_{2} V_{C 00}+b_{3} \bar{V}_{C 11}\right)-\left(1-d^{(1)}\right) V_{C 22}}{(j 2 \omega)^{\alpha} L_{1}} \\
I_{L_{2} 22}=\frac{\left(b_{1} V_{C 11}+b_{2} V_{C 00}+b_{3} \bar{V}_{C 11}\right)-\left(1-d^{(2)}\right) V_{C 22}}{(j 2 \omega)^{\beta} L_{2}}, \\
V_{C 32}=\frac{(1-d)\left(b_{1} I_{L_{1} 22}+b_{2} I_{L_{1} 11}+b_{3} \bar{I}_{L_{1} 00}\right)+(j 3 \omega)^{\beta} L_{2}\left(b_{1} I_{L_{2} 22}+b_{2} I_{L_{2} 11}+b_{3} \bar{I}_{L_{2} 00}\right)}{\Delta(j 3 \omega)}
\end{array}\right.
\end{array}\right.
\end{aligned}
$$




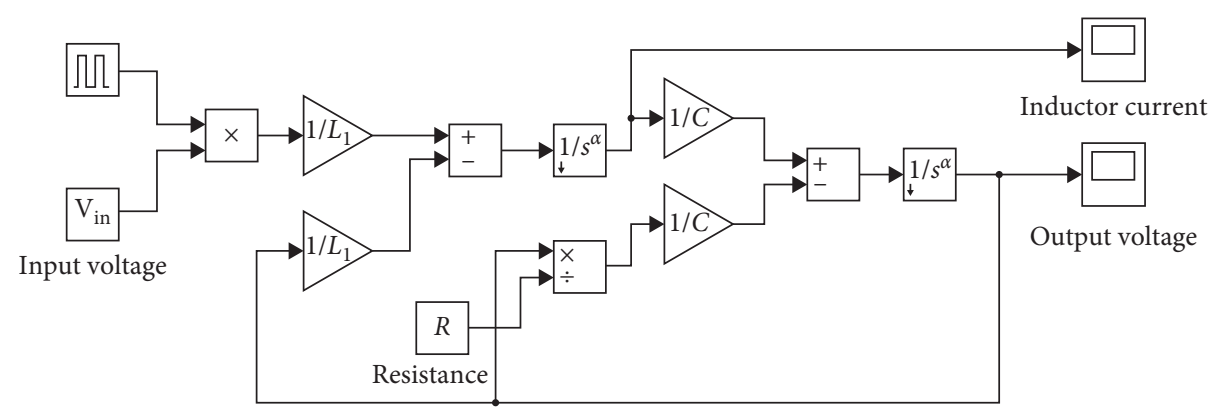

(a)

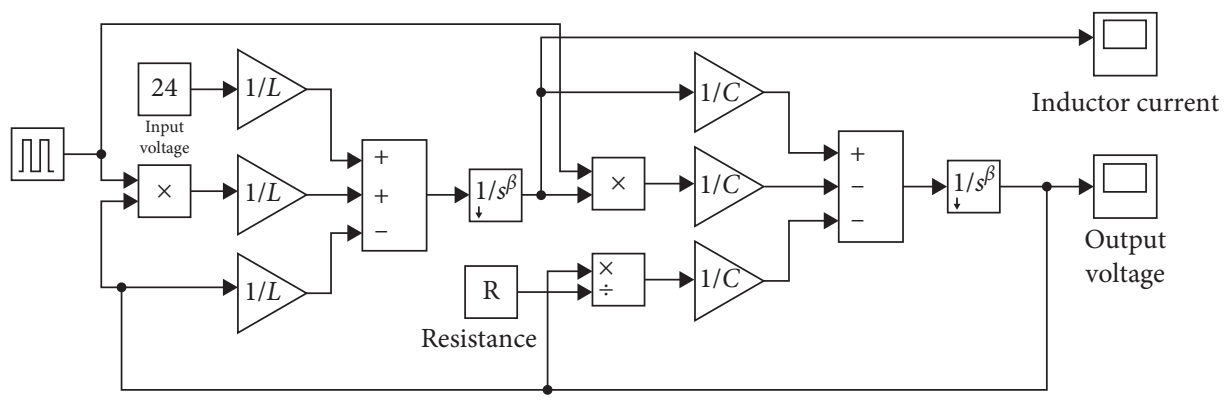

(b)

FIGURE 5: Fractional-order simulation of equivalent DC-DC converter: (a) Buck converter; (b) Boost converter.

Using the principle of harmonic balance, it is possible to approximate $a_{00}, a_{11}, a_{02}, a_{22}, a_{32}$. Thereby, a steady-state periodic solution of the system of equation (25) is obtained, which includes a DC solution and a ripple solution, as

$$
\begin{aligned}
i_{L_{1}} \approx & I_{L_{1} 00}+I_{L_{1} 02}+2\left(\operatorname{ReI}_{L_{1} 11} \cos \tau-\operatorname{Im} I_{L_{1} 11} \sin \tau,\right. \\
& +\operatorname{Re} I_{L_{1} 22} \cos \tau-\operatorname{Im} I_{L_{1} 22} \sin \tau, \\
& +\operatorname{Re} I_{L_{1} 32} \cos \tau-\operatorname{Im} I_{L_{1} 32} \sin \tau, \\
i_{L_{2}} \approx & I_{L_{2} 00}+I_{L_{2} 02}+2\left(\operatorname{ReI}_{L_{2} 11} \cos \tau-\operatorname{Im} I_{L_{2} 11} \sin \tau,\right. \\
& +\operatorname{Re} I_{L_{2} 22} \cos \tau-\operatorname{Im} I_{L_{2} 22} \sin \tau, \\
& +\operatorname{Re} I_{L_{2} 32} \cos \tau-\operatorname{Im} I_{L_{2} 32} \sin \tau, \\
v_{C} \approx & V_{C 00}+V_{C 02}+2\left(\operatorname{Re} V_{C 11} \cos \tau-\operatorname{Im} V_{C 11} \sin \tau,\right. \\
& +\operatorname{Re} V_{C 22} \cos \tau-\operatorname{Im} V_{C 22} \sin \tau, \\
& +\operatorname{Re} V_{C 32} \cos \tau-\operatorname{Im} V_{C 32} \sin \tau .
\end{aligned}
$$

\section{Simulation}

In order to verify the validity and accuracy of the fractionalorder average state space model and the fractional-order equivalent small parameter variable method, simulation studies are implemented in MATLAB2018b [17]. The values of the main parameters of the variable structure HESS $L_{1}, L_{2}$, $C$, and $R$ are set to $100 \mu \mathrm{H}, 100 \mu \mathrm{H}, 470 \mu \mathrm{F}$, and $10 \Omega$, respectively.

According to the fractional average state space model of the equivalent Buck converter and the equivalent Boost converter, the corresponding mathematical simulation models were established in MATLAB2018b/Simulink, as shown in Figure 5. In the open-loop simulation of the equivalent buck converter fractional-order model, the input voltage is set to $24 \mathrm{~V}$, the duty cycle is set to 0.7 , and the order is chosen to be $0.8,0.9$, and 1 , respectively. The simulation results are shown in Figure 6(a) and Figure 6(b). In the openloop simulation of the equivalent Boost converter fractionalorder model, the input voltage is set to $24 \mathrm{~V}$, the duty cycle is set to 0.5 , and the order is chosen to be $0.8,0.9$, and 1 , respectively. The simulation results are shown in Figure 6(c) and Figure 6(d). In addition, experiments were carried out on the Buck mode and Boost mode, respectively. It was found that the experiment results of output voltage are close to the simulation results with the order of 0.95 . This shows that the inductor and capacitor used in the circuit have fractionalorder characteristics, and the order is close to 0.95 .

In order to verify the effectiveness of the fractional-order small parameter variable method for the steady-state analysis of the equivalent dual-switch converter, a simulation program of the fractional-order small parameter variable method was written in MATLAB2018b. The calculation process of programming can be divided into the following 5 parts:

(1) Establish the fractional-order state equation based on the equivalent circuit and determine the sign row vector.

(2) Calculate the Fourier series of the single pulse function.

(3) Calculate the DC component $a_{00}$.

(4) Calculate the ripple components $a_{11}, a_{02}, a_{22}, a_{32}$.

(5) Get the steady-state periodic solution of the equivalent circuit. 


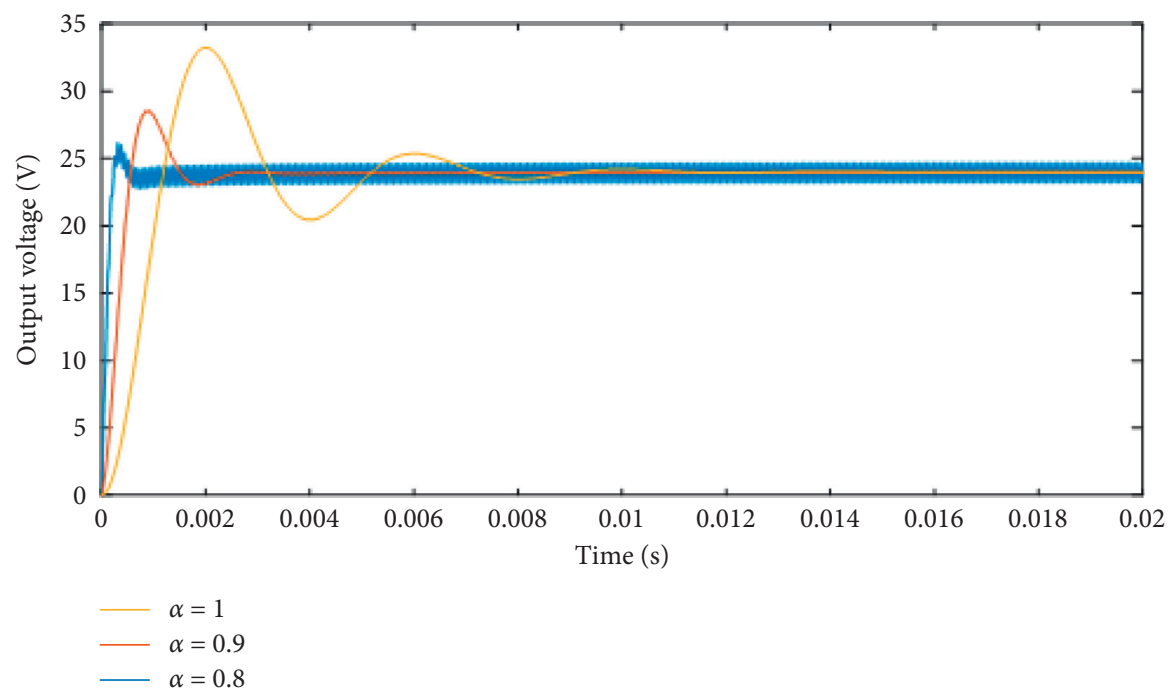

(a)

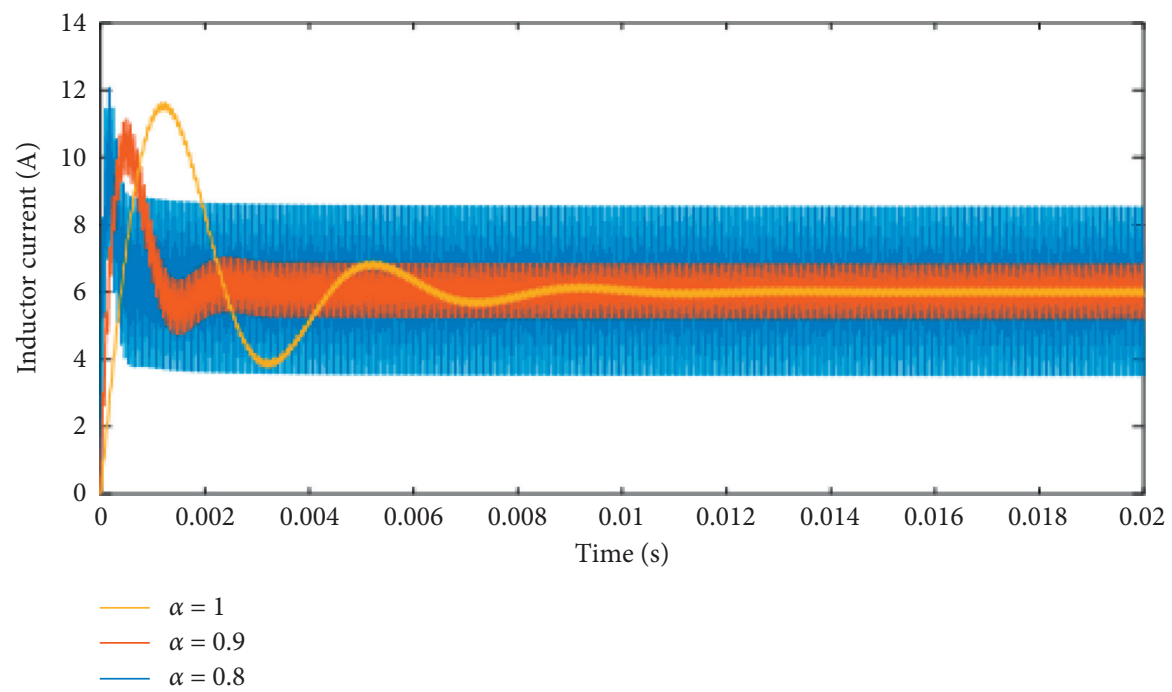

(b)

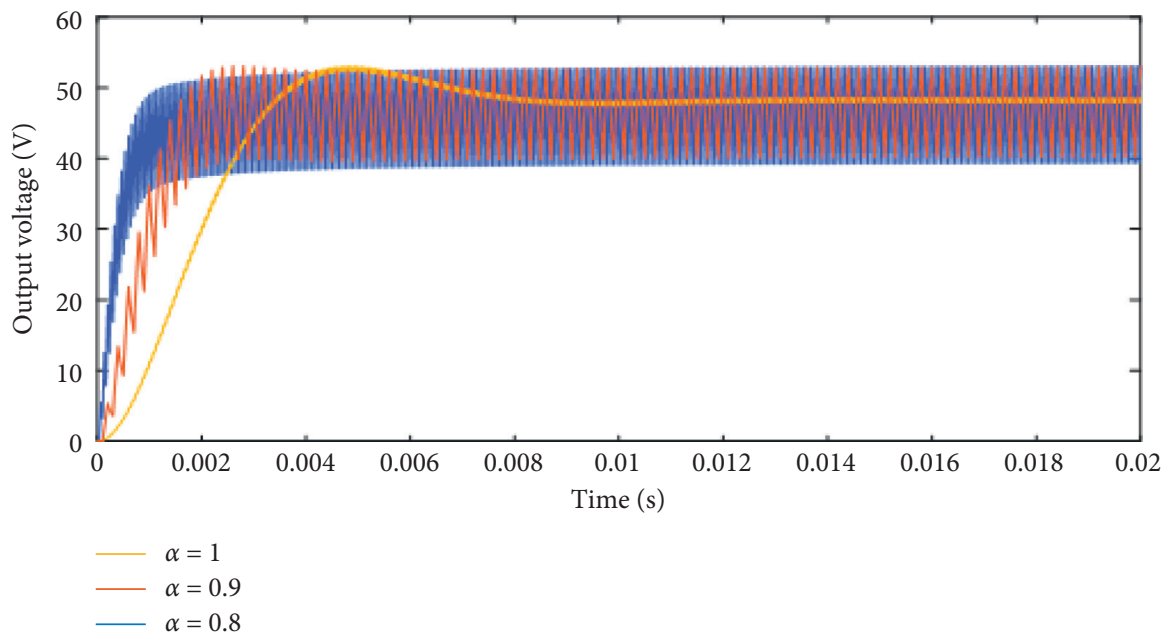

(c)

Figure 6: Continued. 


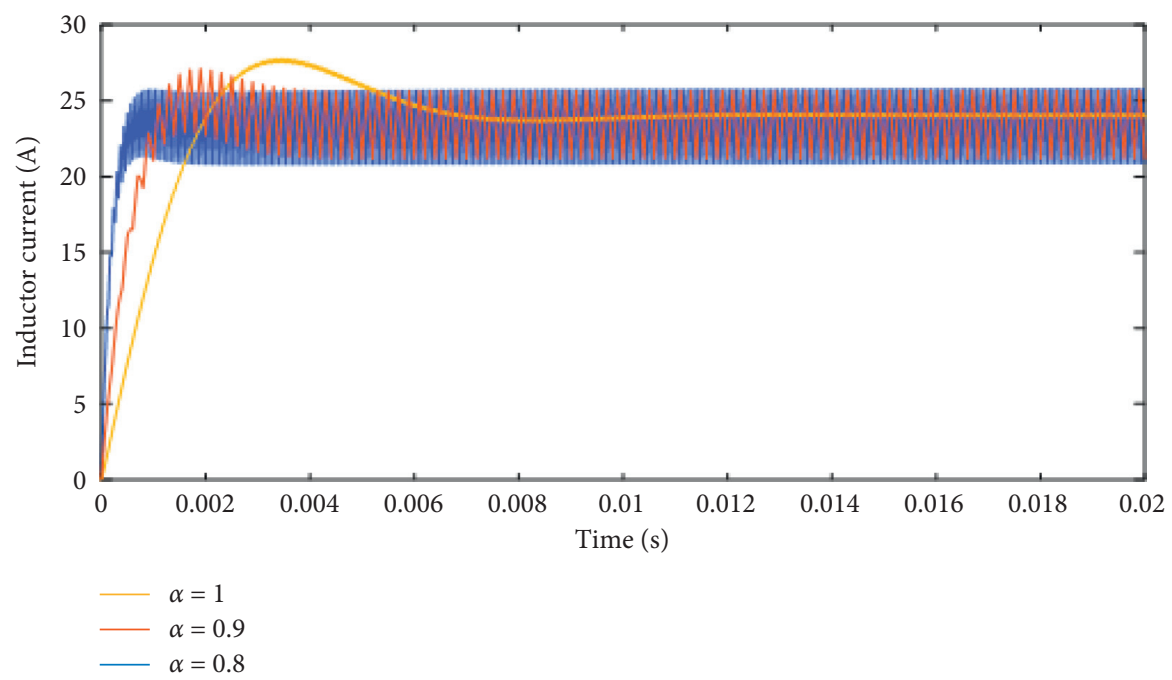

(d)

FIGURE 6: Simulation results of fractional-order model of equivalent DC-DC converter: (a) output voltage of fractional-order model of equivalent Buck converter; (b) inductor current of fractional-order model of equivalent Buck converter; (c) output voltage of fractionalorder model of equivalent Boost converter; (d) inductor current of fractional-order model of equivalent Boost converter.
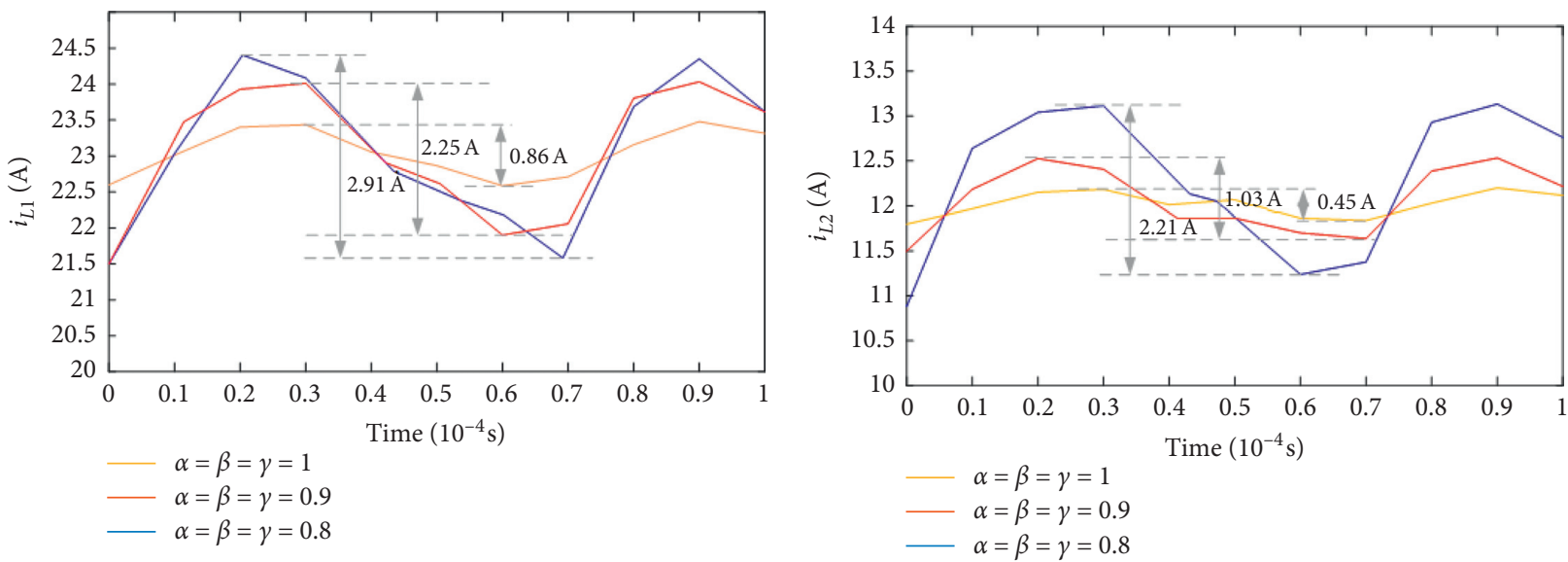

(a)

(b)

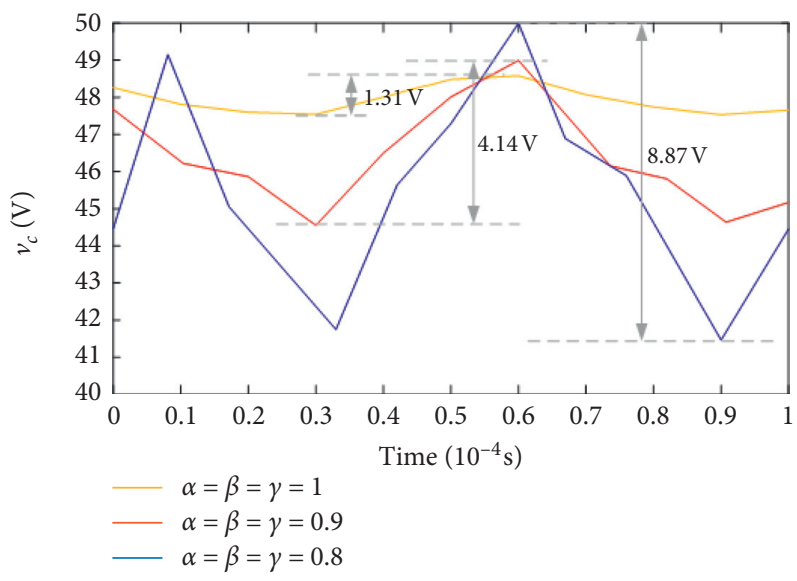

(c)

FIGURE 7: Simulation result of fractional-order model of equivalent dual-switch Boost converter: (a) inductor L1 current; (b) inductor L2 current; (c) output voltage. 
Write (2)-(5) as a subprogram, the main program entry is the circuit parameter matrix and duty cycle row vector, and the exit is the steady-state periodic solution of the circuit. In the simulation process, the SC voltage is set to $38 \mathrm{~V}$, the lithium battery voltage is $24 \mathrm{~V}$, and the duty cycle of SW $\mathrm{SW}_{1}$ and $\mathrm{SW}_{2}$ is set to 0.4 and 0.5 (synchronously boosted to $48 \mathrm{~V}$ output). The calculation results are obtained in the time domain, and the steady-state waveforms that generate the inductor current and output voltage are shown in Figure 7.

From the simulation results, it can be seen that the fractional-order model of the equivalent DC-DC converter has more freedom to choose when describing the DC-DC operating characteristics. As the fractional-order decreases, the overshoot of the dynamic characteristics decreases. The amount of ripple in the steady-state characteristic increases. In practical applications, the voltage response of the equivalent Boost converter of the variable structure HESS has a certain degree of ripple component. However, the ripple of the steady-state characteristics described by the integer-order model is very small, which is obviously in error with the actual situation, and it also shows that the accuracy of integer-order modeling is insufficient. That is to say, when the order of the fractional model is selected to an appropriate value, the dynamic and steady-state characteristics of the DC-DC converter can be accurately described.

\section{Conclusions}

As an important technology for electric vehicles, hybrid energy storage systems have been extensively studied. However, there are still many problems, and this technology cannot be made mature and widely used. Especially the high-precision modeling and control strategy for this complex system is the bottleneck of this technology. In this paper, a fractional-order modeling of a variable structure HESS is carried out. For the three working modes of variable structure HESS, different fractional modeling methods are used to perform fractional modeling and analysis. The effectiveness and precision of the fractional-order model are proved by simulation and experiment.

\section{Data Availability}

The data used to support the findings of this study are available from the corresponding author upon request.

\section{Conflicts of Interest}

The authors declare that they have no conflicts of interest.

\section{Authors' Contributions}

Jianlin Wang and Dan Xu wrote the main manuscript text. Jiahui Zhou and Jinglu Mao assisted in the design of HESS topology and model building. All authors reviewed the manuscript.

\section{References}

[1] M. Sabri, K. Danapalasingam, and M. Rahmat, "A review on hybrid electric vehicles architecture and energy management strategies," Renewable and Sustainable Energy Reviews, vol. 53, pp. 1433-1442, 2016.

[2] J. Cao and A. Emadi, "A new battery/UltraCapacitor hybrid energy storage system for electric, hybrid, and plug-in hybrid electric vehicles," IEEE Transactions on Power Electronics, vol. 27, pp. 122-132, 2012.

[3] Z. Song, H. Hofmann, J. Li, X. Han, X. Zhang, and M. Ouyang, "A comparison study of different semi-active hybrid energy storage system topologies for electric vehicles," Journal of Power Sources, vol. 274, pp. 400-411, 2015.

[4] H. S. Das, C. W. Tan, and A. H. M. Yatim, "Fuel cell hybrid electric vehicles: a review on power conditioning units and topologies," Renewable and Sustainable Energy Reviews, vol. 76, pp. 268-291, 2017.

[5] M. B. Camara, H. Gualous, F. Gustin, A. Berthon, and B. Dakyo, "DC/DC converter design for supercapacitor and battery power management in hybrid vehicle applicationspolynomial control strategy," IEEE Transactions on Industrial Electronics, vol. 57, no. 2, pp. 587-597, 2010.

[6] C. Ren, X. S. He, and H. R. F. KarimiLiu, "Finite-time L2-Gain asynchronous control for continuous-time positive hidden Markov jump systems via T-S fuzzy model approach," IEEE Transactions on Cybernetics, vol. 99, pp. 1-11, 2020.

[7] C. Ren and S. He, "Finite-time stabilization for positive Markovian jumping neural networks," Applied Mathematics and Computation, vol. 365, Article ID 124631, 2020.

[8] C. Ren, R. Nie, and S. He, "Finite-time positiveness and distributed control of Lipschitz nonlinear multi-agent systems," Journal of the Franklin Institute, vol. 356, no. 15, pp. 8080-8092, 2019.

[9] A. Davoudi and J. Jatskevich, "Realization of parasitics in state-space average-value modeling of PWM DC-DC converters," IEEE Transactions on Power Electronics, vol. 21, no. 4, pp. 1142-1147, 2017.

[10] J. Wang, D. Xu, H. Zhou, and A. Bai, "High-performance fractional order terminal sliding mode control strategy for DC-DC Buck converter," PLoS One, vol. 12, no. 10, Article ID e0187152, 2017.

[11] C. Wu, G. Si, Y. Zhang, and N. Yang, "The fractional-order state-space averaging modeling of the Buck-Boost DC/DC converter in discontinuous conduction mode and the performance analysis," Nonlinear Dynamics, vol. 79, no. 1, pp. 689-703, 2015.

[12] H. Komurcugil, "Adaptive terminal sliding-mode control strategy for DC-DC buck converters," ISA Transactions, vol. 51, no. 6, pp. 673-681, 2012.

[13] G.-C. Wu, D. Baleanu, and W.-H. Luo, "Lyapunov functions for Riemann-Liouville-like fractional difference equations," Applied Mathematics and Computation, vol. 314, pp. 228-236, 2017.

[14] I. M. Filanovsky and S. S. Shui-Sheng Qiu, "Harmonic analysis of PWM converters," IEEE Transactions on Circuits and Systems I: Fundamental Theory and Applications, vol. 47, no. 9, pp. 1340-1349, 2000.

[15] A. G. Radwan, A. A. Emira, A. M. Abdelaty et al., "Modeling and analysis of fractional order DC-DC converter," ISA Transactions, vol. 82, pp. 184-199, 2017.

[16] X. Chen, Y. F. Chen, B. Zhang et al., "A method of modeling and analysis for fractional-order DC-DC converters," IEEE Transactions on Power Electronics, vol. 99, 2016.

[17] J. L. Wang, L. Zhang, J. L. Mao et al., "Fractional order equivalent circuit model and SOC estimation of supercapacitors for use in HESS," Digital Object Identifier, vol. 10, Article ID 2912221, 2019. 